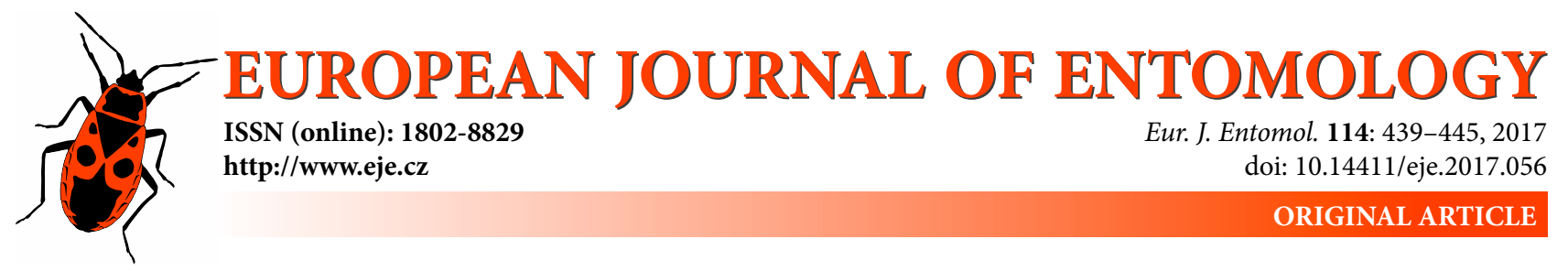

\title{
Gut microbiota influences female choice and fecundity in the nuptial gift-giving species, Drosophila subobscura (Diptera: Drosophilidae)
}

\author{
Benjamin S. WALSH, Chloe HeYS and Zenobia LeWiS* \\ School of Life Sciences/Institute of Integrative Biology, University of Liverpool, Crown Street, L69 7ZB Liverpool, UK; \\ e-mails: bwalsh@liverpool.ac.uk, bs0u911f@liverpool.ac.uk, z.lewis@liverpool.ac.uk
}

Key words. Diptera, Drosophilidae, Drosophila subobscura, microbiota, gut bacteria, mate preference, reproductive success

\begin{abstract}
Recently, there has been rapidly growing interest in the effects of the microbiota on host physiology and behaviour. Due to the nutritional value of bacteria, gut microflora may be particularly important in species that present nuptial gifts during courtship. Here, we explore whether the presence or absence of gut microbiota in males and females of the nuptial gift-giving species Drosophila subobscura (Collin, 1936) alters mating behaviour in terms of female preference, male investment, and female fecundity. We found that females that had been fed antibiotics, compared to females with intact gut bacteria, were more willing to mate with a male that had been fed normally. However female fecundity was higher when both males and females lacked gut bacteria compared to both individuals having a full complement of gut bacteria. This implies that the presence of the microbiota acts to reduce female fecundity in this species, and that male gut bacterial content influences female fecundity. Our results provide further evidence to the growing consensus that the microbiota of an individual may have important effects on both reproductive behaviour and physiology, and suggest that it may also contribute to the nutritional value of the nuptial gift in this system.
\end{abstract}

\section{INTRODUCTION}

Recently, there has been rapidly growing interest in the effects of the microbiota: the parasitic, commensal, and symbiotic microorganisms residing within and on their host (reviewed in Lewis \& Lizé, 2015). The community of bacteria residing in the gut of the host has, in particular, received attention as it has been shown to have important effects on the fitness (e.g. Rosengaus et al., 2011), behaviour (e.g. Sharon et al., 2010), and evolution (e.g. Brucker $\&$ Bordenstein, 2013) of the host. In insects, bacterial communities of one individual are known to alter behaviour of others, through communicating essential information from the host through changes in host odour, primarily through altering the cuticular hydrocarbon $(\mathrm{CHC})$ profile (Sharon et al., 2010). For example, gut bacteria plays an important role in masking an inherent kin recognition mechanism in the fruit fly Drosophila melanogaster, potentially through olfactory cues (Lizé et al., 2014). When naturally occurring gut bacteria in D. melanogaster are removed, males transfer less sperm to related females as compared to unrelated females, which reduces the potential costs of inbreeding. This suggests the presence of a naturally occurring kin recognition ability that is suppressed by gut bacteria. Thus, it is of great importance to examine any other potential physiological and behavioural effects that commensal gut bacteria can have on its host species. One area that remains underexplored with regards to the microbiome and both mating behaviour and physiology is the potential link between microbes in the gut, and the composition of nuptial gifts.

In some insect species, males present females with edible nuptial gifts before, during, or after copulation. These gifts can take the form of collected food items, or other tokens such as glandular secretions, nutritious spermatophores, or even self-sacrifice (Thornhill \& Alcock, 1983). The benefits nuptial gifts provide to females are relatively transparent - consuming the food item usually provides females with important nutrients that can be used for immediate benefit. As beneficial bacteria are shown to increase the nutritional value of a food product (e.g. Drew et al., 1983; Zhang et al., 2005; Pradel et al., 2007), it can therefore be suggested that, gut bacteria could impact the value of a nuptial gift to a potential mate, through changing the nutritional quality, or through changes in host odour. This could influence mate choice or sexual selection (e.g. Everaerts et al., 2010; Scott et al., 2011; Sharon et al., 2011). Therefore, not only can the gut bacterial content of an individual's gut influence the physiology and behaviour of its host, but it may convey important cues to potential mates, which may alter the mating behaviour of others. Indeed,

\footnotetext{
* Corresponding author; e-mail: z.lewis@liverpool.ac.uk
} 
it has been shown in the fruit fly Ceratitis capitata that removing gut bacteria results in an increase in oviposition rate, although it should be noted that overall fecundity remained consistent regardless of gut bacterial content (BenYosef et al., 2008). This study suggests that gut bacterial content may have important behavioural and physiological effects on an individual. It is therefore possible that the gut bacterial content of a nuptial gift itself may contribute to these effects. To understand the way in which nuptial gift-giving behaviour influences the behaviour and physiology of males and females, we need to examine in more detail the components which constitute the gift. In some nuptial gift-giving species females can assess the quality and/or size of a nuptial gift, and will base their preference of males on this assessment (Albo et al., 2013). In light of this, what components of a nuptial gift contribute to its physiological benefit, and how can females judge this quality, preferentially choosing "better" gifts?

Males of the fruit fly Drosophila subobscura (Collin, 1936) provide a nuptial gift during courtship; a droplet is secreted from the male's crop and presented to the female on his proboscis (Krimbas, 1993). Similar to many other nuptial gifts, the nutritional significance of this gift has been shown to be an important factor in the mating behaviour of this species. It has already been demonstrated in D. subobscura that starved females are more likely to choose well fed males, and the fecundity of these females is higher when they do (Immonen et al., 2009). When well fed, males produce larger gifts at a greater quantity, which is believed to contribute to this increase in fecundity. Although this may be because males in good "condition" produce a greater quantity of drops, it could also indicate that females possess the ability to assess drop quality, and females may do this using olfactory cues. Although the importance of this gift has already been outlined, what constitutes quality of the gift has not yet been demonstrated. As an individual's gut bacterial content can have such important effects on host physiology and mating behaviour, and is highly likely to be present in the gift itself, this could also impact both nuptial gift quality and its olfactory cues.

The first aim of this study was to evaluate whether the presence or absence of a full complement of gut bacteria affects female preference in D. subobscura. The courtship experiment utilised a between-subjects two-by-two design. Individuals were either fed normally, and therefore had a "typical" gut bacterial content, or they were fed with antibiotic-supplemented food. Adding antibiotics to the rearing medium is a standard method of suppressing gut bacteria (Koukou et al., 2006; Ben-Yosef et al., 2008; Sharon et al., 2010; Lizé et al., 2014). However, in order to confirm that our technique resulted in suppression of the gut microbiota, we analysed the bacterial content of the $D$. subobscura gut from both the normal and the streptomycin reared diets. Normally-fed males were paired with either normally-fed or antibiotic-supplemented females, and similarly, antibiotic-supplemented males were paired with either normally-fed or antibiotic-supplemented females. We predicted that antibiotic-supplemented females, com- pared to normally-fed females, would be more willing to mate with normally-fed males, if gut bacteria contributes to the nutritional importance of the gift as shown by Immonen et al. (2009). Ideally, this study would involve directly manipulating the gift, and feeding females gifts from experimentally treated males before mating with control males in order to control for any other potential effects of the treatment on males, such as sperm count. However this would be almost impossible due to the small size of the gifts, and the fact that the gift is so vital to the courtship of this species, and it is likely that any control males would also give nuptial gifts to the females, potentially transferring bacteria to the female whilst doing so.

The second aim of this study was to evaluate whether the bacteria residing within the male and/or female affects the female's fecundity after accepting a nuptial gift. We predicted that if the gut and/or crop bacteria are beneficial to $D$. subobscura, antibiotic-supplemented females should have lower fecundity when mated with antibioticsupplemented males, but if the gut bacterial content negatively impacts the hosts, that the removal of this microbiota would actually have a positive impact on the host.

\section{MATERIALS AND METHODS}

\section{Experimental flies}

Stocks were obtained from Punta Umbria, Spain $\left(37.10^{\circ} \mathrm{N}\right.$, $6.57^{\circ} \mathrm{W}$ ) in April 2013, and reared in vials containing $20 \mathrm{ml}$ standard maize-sugar-yeast agar "ASG" medium. Flies were reared at $18^{\circ} \mathrm{C}$ and on a $12 \mathrm{~L}: 12 \mathrm{D}$ cycle. To obtain experimental flies, flies from this stock were separated at eclosion and mated after 7 days, when sexual maturity is reached in this species. The adults were then mated in a cylindrical mating chamber of height $12.5 \mathrm{~cm}$ and diameter $10 \mathrm{~cm}$, and left for $48 \mathrm{~h}$ to oviposit on an agar plate on the bottom containing grape juice medium (made by mixing $12.5 \mathrm{~g}$ agar, $275 \mathrm{ml}$ water, $150 \mathrm{ml}$ grape juice, and $10.5 \mathrm{ml}$ of nipagin). Larvae were subsequently transferred from the agar plate to one of the two different food treatment types: (1) ASG double yeast medium (made by mixing $40 \mathrm{~g}$ agar, $340 \mathrm{~g}$ sugar, $240 \mathrm{~g}$ maize, $160 \mathrm{~g}$ yeast, $4 \mathrm{~L}$ water and $100 \mathrm{ml}$ nipagin together) and (2) ASG double yeast medium supplemented with the antibiotic streptomycin (using the same recipe as before, but adding $4 \mathrm{ml}$ of $0.04 \%$ streptomycin dissolved in ethanol per litre food). This results in a very small discrepancy in ethanol between the two treatments, however due to the small quantity involved, it is unlikely that this will have had any effect. Flies were transferred into vials at a density of 10 larvae per vial, thereby controlling for any potential density effects. Larvae were then left to mature and at eclosion, virgin adults were anaesthetised and separated into individual vials containing the same food type that they were reared on as larvae.

\section{Confirmation of suppressed gut microbiota}

In order to ensure that the addition of streptomycin $(0.04 \%$ concentration) to the dietary media did indeed supress gut bacterial communities, we analysed the bacterial content of the $D$. subobscura gut from both treatments. The whole gut of seven day-old flies was dissected into either $500 \mu \mathrm{l}$ of PBS or $500 \mu \mathrm{l}$ of liquid Brain Heart Infusion (BHI) media. These two dissection mediums and two growth mediums [BHI and MRS (de Man, Rogosa and Sharpe) agar] were used in order to ensure that a particular media did not select for certain species, thereby ensuring that any bacterial growth present would be representative of the 


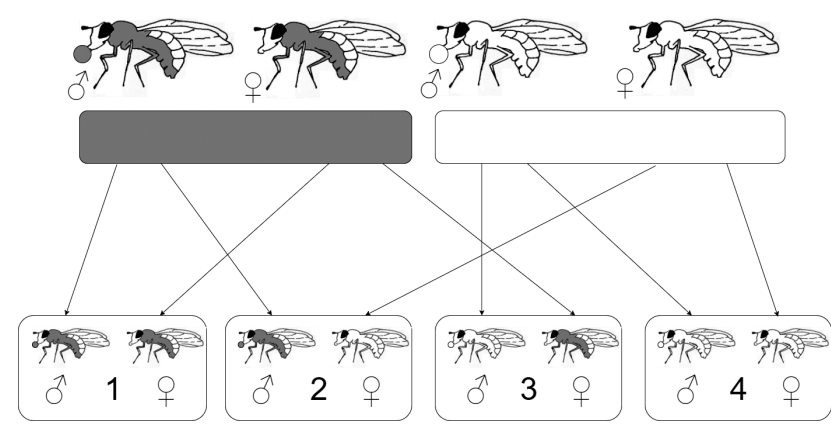

Fig. 1. A diagram outlining the different mating treatments used in the study. Males are shown as flies with a round circle, representing the nuptial gift, connected to their proboscis, and females without. Shaded flies represent flies that have not been treated with antibiotic and therefore have gut bacteria present, and white flies represent flies fed on antibiotics.

D. subobscura gut. Dissection was repeated three times in both media for each treatment type: normal flies and flies reared on a streptomycin diet. The gut-solute was then placed into a $1.5 \mathrm{ml}$ Eppendorf and disrupted by hand using a sterile pestle. $100 \mu \mathrm{l}$ of the solute was then transferred to the centre of both MRS and BHI agar plates. The solute was spread across the whole plate with a sterile glass loop and sealed, before being placed into an incubator at $25^{\circ} \mathrm{C}$ for $72-96 \mathrm{~h}$. After this time, the plates were checked for bacterial growth, and the number of colonies counted.

\section{Mating trials}

7 days after eclosion, the experimental adults were mated. Matings were conducted at $21^{\circ} \mathrm{C}$, and started at $10 \mathrm{am}$. A bar light was placed under the shelves to maximise light which is vital to successful matings in D. subobscura (Krimbas, 1993). Fig. 1 outlines the different mating treatments, comprising males and females that were reared on $20 \mathrm{ml}$ of either normal ASG medium, or streptomycin antibiotic medium. Sample sizes were as follows: treatment $1=36$, treatment $2=33$, treatment $3=36$, and treatment $4=33$.

Males were aspirated into vials, each of which already contained a female. Pairs were watched for an hour or until the end of copulation. The time that copulation started and copulation duration were measured. Mating success was measured as the success/failure to copulate during the $1 \mathrm{~h}$ session. Mating latency, a commonly-used measure of female preference as it is driven by females themselves (Bacigalupe et al., 2007; Debelle et al., 2014), was taken as the time (measured in seconds) from the placement of the male into the vial containing the female, until the beginning of copulation. Copulation duration, a commonly used measure of male investment as it is driven by males (Harcourt et al., 1981; Jennions \& Passmore, 1993; Price et al., 2012) was calculated as the copulation time from beginning to end (also measured in seconds). Mating latency and copulation duration are commonly used indicators of mate preference and mating investment, respectively, in Drosophila (e.g. Verspoor et al., 2015).

\section{Measuring fecundity}

Following mating, females were transferred into a vial for oviposition. The vial contained $20 \mathrm{ml}$ of neutral grape juice medium and a $1.5 \mathrm{~cm}$ centrifuge tube containing yeast paste, with small holes pierced near the top, ensuring that the yeast was inaccessible to the female for feeding, but would encourage ovipositing through olfactory cues. Female fecundity was taken as the number of eggs oviposited onto this grape juice medium sampled at two specific times: for a $48 \mathrm{~h}$ period directly after mating and another $48 \mathrm{~h}$ period 7 days after mating. In between these two egg collection episodes, the flies were kept alone in $20 \mathrm{ml}$ of neutral grape-juice medium. This allowed us to have a total measure of fecundity, further split into both short term and long term fecundity, to examine whether the effects of the treatments changed over time.

\section{Statistical analyses}

To examine whether antibiotic treatment affected mating success $(0 / 1)$, a logistic regression was used. This logistic regression was conducted in R v3.4.0 (R Core Team, 2015). A fully saturated model that included male treatment, female treatment and their interaction, was reduced to a minimum adequate model through step-wise simplification and Wald $\mathrm{Chi}^{2}$ tests with the "car" package. Effect sizes were obtained from predictor variable covariates of the final model. Models were assessed for over-dispersion and the fit was verified through inspecting patterns in simulated residuals generated with the "DHARMa" package (Hartig, 2017).

The response variables were log-transformed after finding that none [latency (s), mating duration (s), fecundity straight after mating, and fecundity 7 days after mating, total fecundity] fit a normal distribution. We then tested for differences in these variables across treatment groups using a multi-factorial general linear model with male and female treatment type as the two factors. The statistical software SPSS (v22) was used for these general linear models (IBM Corp, 2013).

\section{RESULTS}

\section{Suppression of gut microbiota}

On both the growth mediums, significantly more bacterial colonies grew from flies with a normal gut complement. In most cases from flies that had been reared on a streptomycin diet there were zero colonies present (Table 1), suggesting that the gut microbiota was completely suppressed. There was slight bacterial growth on four of the

Table 1. The number of colonies grown on two different growth mediums - BHI (Brain Heart Infusion) and MRS (de Man, Rogosa and Sharpe) - and the solution the gut was dissected into. "Antibiotic" and "normal" refer to the antibiotic-supplemented ASG medium and normal ASG double yeast medium respectively, that the individuals were reared on. Some replicates were removed from the analysis due to mould contamination.

\begin{tabular}{ccccc}
\hline Diet type & Replicate & $\begin{array}{c}\text { Dissection } \\
\text { solution }\end{array}$ & Media & $\begin{array}{c}\text { Number of } \\
\text { colonies }\end{array}$ \\
\hline Normal & 1 & PBS & BHI & 1976 \\
Normal & 2 & PBS & BHI & 624 \\
Normal & 3 & PBS & BHI & 16 \\
Normal & 1 & PBS & MRS & 1612 \\
Normal & 2 & PBS & MRS & 728 \\
Normal & 3 & PBS & MRS & 17 \\
Normal & 1 & BHI & MRS & 1924 \\
Normal & 2 & BHI & MRS & 5512 \\
Normal & 3 & BHI & MRS & 9100 \\
Normal & 1 & BHI & BHI & 3068 \\
Normal & 2 & BHI & BHI & 3120 \\
Antibiotic & 1 & PBS & MRS & 0 \\
Antibiotic & 2 & PBS & MRS & 0 \\
Antibiotic & 3 & PBS & MRS & 0 \\
Antibiotic & 2 & BHI & MRS & 0 \\
Antibiotic & 3 & BHI & MRS & 0 \\
Antibiotic & 1 & BHI & BHI & 1 \\
Antibiotic & 2 & BHI & BHI & 0 \\
Antibiotic & 3 & BHI & BHI & 1 \\
Antibiotic & 2 & PBS & BHI & 1 \\
Antibiotic & 3 & PBS & BHI & 11 \\
\hline
\end{tabular}




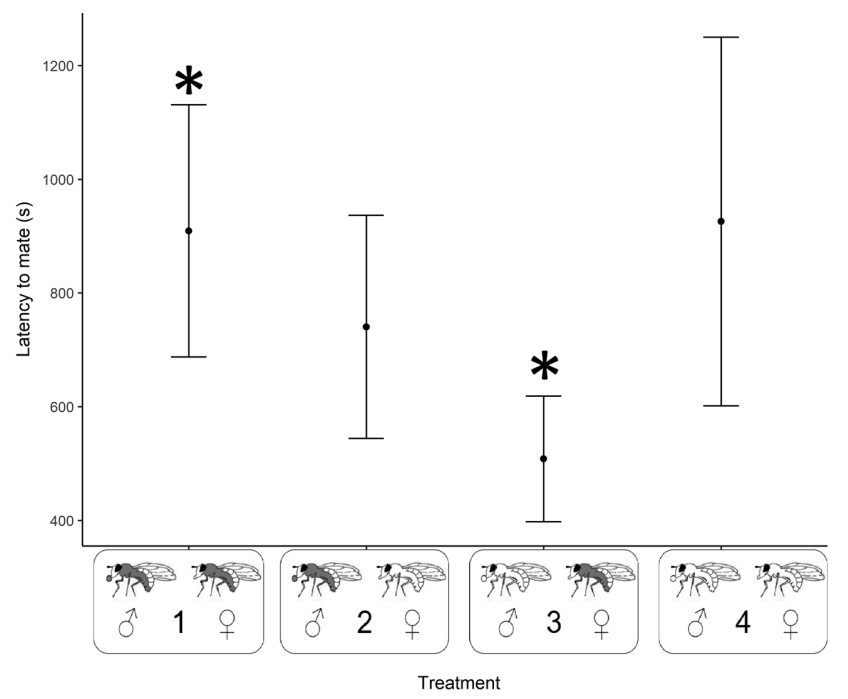

Fig. 2. The mating latency of $D$. subobscura. Normally-fed males were paired with either normally-fed (1) or antibiotic-supplemented females (3), and similarly, antibiotic-supplemented males were paired with either normally-fed (2) or antibiotic-supplemented females (4). Standard error bars are included. There was a significant interaction between male and female treatment, indicated by the stars above the standard error bars. Females mated faster when they had been fed antibiotics, and were presented with a male which had been fed normally. Here, the raw data are presented but for the purpose of analysis, the data for mating latency were log-transformed. Diagram created in the statistical software SPSS.

ten plates containing flies that had been reared on a streptomycin diet, potentially as a result of contamination. In these cases however, the colony count was much lower,

Table 2. Means \pm standard deviations for the response variables analysed. "Antibiotic" and "normal" refer to the antibiotic-supplemented ASG double yeast medium and normal ASG double yeast medium respectively, that the individuals were reared on. Stars indicate treatments with statistically significantly differences with each other $(p<0.05)$. Fecundity A and fecundity B are the fecundity measures taken straight after mating, and after 7 days, respectively.

\begin{tabular}{|c|c|c|c|c|}
\hline $\begin{array}{l}\text { Response } \\
\text { variable }\end{array}$ & $\begin{array}{c}\text { Treatment } \\
\text { number }\end{array}$ & $\begin{array}{c}\text { Male } \\
\text { treatment }\end{array}$ & $\begin{array}{l}\text { Female } \\
\text { treatment }\end{array}$ & $\begin{array}{c}\text { Mean } \pm \text { standard } \\
\text { deviation }\end{array}$ \\
\hline \multirow{4}{*}{$\begin{array}{c}\text { Mating } \\
\text { latency (s) }\end{array}$} & $\mathrm{T}^{*}{ }^{*}$ & Normal & Normal & $909.09 \pm 645.59$ \\
\hline & $\mathrm{T} 2$ & Antibiotic & Normal & $734.52 \pm 558.31$ \\
\hline & $\mathrm{T}^{*}$ & Normal & Antibiotic & $508.00 \pm 316.78$ \\
\hline & $\mathrm{T} 4$ & Antibiotic & Antibiotic & $917.15 \pm 803.63$ \\
\hline \multirow{4}{*}{$\begin{array}{c}\text { Mating } \\
\text { duration (s) }\end{array}$} & T1 & Normal & Normal & $383.80 \pm 135.01$ \\
\hline & T2 & Antibiotic & Normal & $407.48 \pm 236.34$ \\
\hline & T3 & Normal & Antibiotic & $377.44 \pm 172.12$ \\
\hline & T4 & Antibiotic & Antibiotic & $392.54 \pm 134.99$ \\
\hline \multirow{4}{*}{ Fecundity A } & T1 & Normal & Normal & $10.08 \pm 13.07$ \\
\hline & T2 & Antibiotic & Normal & $6.20 \pm 9.79$ \\
\hline & T3 & Normal & Antibiotic & $8.44 \pm 10.78$ \\
\hline & T4 & Antibiotic & Antibiotic & $14.78+13.52$ \\
\hline \multirow{4}{*}{ Fecundity B } & T1 & Normal & Normal & $9.07 \pm 11.39$ \\
\hline & $\mathrm{T} 2$ & Antibiotic & Normal & $15.35 \pm 13.69$ \\
\hline & T3 & Normal & Antibiotic & $11.20 \pm 10.60$ \\
\hline & $\mathrm{T} 4$ & Antibiotic & Antibiotic & $15.86 \pm 12.58$ \\
\hline \multirow{4}{*}{$\begin{array}{c}\text { Total } \\
\text { fecundity }\end{array}$} & T1* & Normal & Normal & $17.80 \pm 21.92$ \\
\hline & $\mathrm{T} 2$ & Antibiotic & Normal & $20.67 \pm 18.37$ \\
\hline & T3 & Normal & Antibiotic & $18.88 \pm 17.98$ \\
\hline & $\mathrm{T} 4^{*}$ & Antibiotic & Antibiotic & $28.20 \pm 14.77$ \\
\hline
\end{tabular}

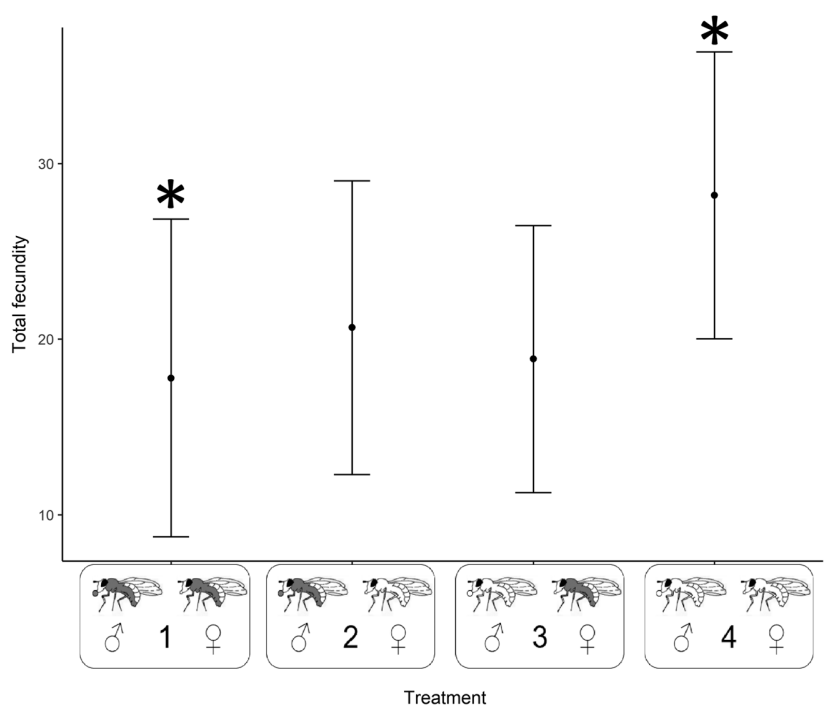

Fig. 3. The total fecundity of $D$. subobscura. Normally-fed males were paired with either normally-fed (1) or antibiotic-supplemented females (3), and similarly, antibiotic-supplemented males were paired with either normally-fed (2) or antibiotic-supplemented females (4). Standard error bars are included. The addition of antibiotics to the food of both males and females significantly increased female fecundity, with significant differences indicated by the stars above the standard error bars. Here, the raw data are presented but for the purpose of analysis, the data for total fecundity were log-transformed. Diagram created in the statistical software SPSS.

and sufficiently shows that our treatment types were effective in suppressing gut microbiota.

\section{Mating success}

The minimum adequate model of mating success retained only female treatment as a significant predictor (Wald $\chi^{2}$, d.f. $=1, P=0.030$ ). Females treated with antibiotics had a +2.322 increase in log odds of mating compared to normally treated females, and thus are more likely to be mated. However, one should be cautious when interpreting this result, as only 1/69 microbiota-free, and 9/69 normal flies failed to mate and so this is testing close to the boundary of the data.

\section{Latency}

There was no main effect of male treatment on mating latency $(F=0.08$, d.f. $=3, p=0.785)$. There was also no main effect of female treatment on mating latency $(F=$ 0.49 , d.f. $=1, p=0.487$ ). However there was a statistically significant interaction between male treatment and female treatment affecting mating latency $(F=5.68$, d.f. $=1, p=$ $0.020)$. Females that had their gut microbiota suppressed mated faster with males that had an intact microbiota, as shown by Fig. 2.

\section{Mating duration}

There was no main effect of male $(F=0.53$, d.f. $=1, p=$ $0.470)$ or female treatment $(F=0.20$, d.f. $=1, p=0.653)$ on mating duration. There was also no interaction between these factors $(F=0.30$, d.f. $=1, p=0.586)$. 


\section{Fecundity}

When examining total fecundity, there were statistically significant effects of the two factors, as shown by Fig. 3 . Female fecundity was higher when the female was raised on antibiotics $(F=4.48$, d.f. $=1, p=0.037)$, and when the male was raised on antibiotics $(F=4.95$, d.f. $=1, p=$ 0.029).

When the data was split into short term and long term fecundity, there were no main effects of either male $(F=$ 0.17 , d.f. $=1, p=0.682)$ or female $(F=1.59$, d.f. $=1, p=$ $0.211)$ treatment on immediate fecundity, nor was there an interaction between these factors $(F=2.02$, d.f. $=1, p=$ 0.159). Similarly, there were no main effects of either male $(F=3.50$, d.f. $=1, p=0.065)$ or female $(F=2.47$, d.f. $=1$, $p=0.120)$ treatment on fecundity a week after copulation, nor was there an interaction between these factors $(F=$ 0.19 , d.f. $=1, p=0.661$ ).

\section{DISCUSSION}

This study suggests that gut bacteria can have an important impact on both the behaviour and the physiology of Drosophila subobscura. We found that female treatment had a significant impact on their mating behaviour. Females that had their gut microbiota suppressed mated faster with males that had an intact microbiota, whereas females with an intact gut microbiota were less willing to mate with males with an intact microbiota. Microbiota-free females were more likely to mate than normal females. It is likely that this result is an artefact of their lower mating latency; microbiota-free females mate more readily, and are therefore more likely to be mated at all in the $1 \mathrm{~h}$ observation window. There were no differences in male investment across treatments, when measured as mating duration. Total fecundity was increased by removing the gut bacteria of both the male and the female flies, as compared to total fecundity when both the male and female were fed normally. Our findings suggest that there may be a link between gut bacteria and the mating behaviour of D. subobscura. The fact that we found female treatment to have an impact on mating latency, suggests that the presence or absence of gut bacteria in males can be detected by females, and that this alters females behaviour. Specifically, we found that removing the female's gut microflora made them more willing to mate with a normally-fed male, which suggests that they were in a worsened state (Immonen et al., 2009) However, the fecundity of a female was actually lower if both partners were normally-fed, suggesting that the bacteria in the gut actually may act to reduce female fecundity.

A difficult question arises from these results. If treated females more readily choose to mate with a male that has intact gut bacteria, one could predict that the preference may benefit females by allowing them to choose a "better quality" gift from a normally fed male's gift; it has already been shown that starved females of $D$. subobscura exhibit a similar response (Immonen et al., 2009). However as this female preference does not result in increased fecundity, for what reason do females more readily mate with normally-fed males when the female lacks gut bacteria?
One possible reason as to why females are willing to mate is that they are in a weakened state, making them less able to resist mating attempts from males. It is unclear whether this is happening here, because mating latency is femaledriven in this species: when the male is rejected the female orients its ovipositor downward, which makes it difficult for the male to copulate (Krimbas, 1993). However, just because they are capable of resisting males, the impacts of male harassment on female behaviour may differ between the treatment types.

A similar study examining the fruit fly C. capitata showed that removing the commensal gut bacteria results in no difference of fecundity but an increase in oviposition rate (Ben-Yosef et al., 2008). Although this effect is slightly different to the one we have shown in D. subobscura as overall fecundity is not shown to be different, it reaffirms that bacteria must play an important part in post-mating female reproductive physiology and behaviour. It should also be noted that the initial increase in oviposition when bacteria are absent in $C$. capitata asks similar questions about whether the "commensal" bacteria in the gut is actually having adverse effects on its host.

The fact that antibiotic supplementation resulted in increased female fecundity is curious. Indeed, previous studies on the physiological effects of disruption of the microbiome in Drosophila species have shown contrasting results. Generally, disruption of host microbiome has resulted in negative physiological effects, including increased larval development time, as well as reduced protein content (Ridley et al., 2012; Wong et al., 2014). These studies also failed to find an effect of microbiome status on fecundity; however it is notable that microbial effects on Drosophila are not uniform between males and females, which is supported by our results (Wong et al., 2014).

The question of why the disruption of microbiota leads to an increase in fecundity in our study may be explained in an alternative way; potentially deleterious endosymbionts within the host cells are suppressed by the antibiotics, resulting in an increase in fecundity. It has been shown that removal of the endosymbiont Wolbachia in American populations of Drosophila suzukii results in an increase in fecundity compared to infected individuals, so a similar scenario may be present in this study (Hamm et al., 2014). However, in a European population of D. suzukii, removal of Wolbachia actually results in a decrease in fecundity (Mazzetto et al., 2015). Thus, even within the same Drosophila species, there are contrasting effects of Wolbachia on host fitness, so it is therefore unlikely to be as a result of Wolbachia. Additionally, to our knowledge, Wolbachia have not yet been found in D. subobscura (Haine et al., 2005). Potentially the D. subobscura utilised in the current study harbour a different endosymbiont that is mediating the effect we have found, and as such it may be of importance to examine the presence or absence of such endosymbionts in future studies.

We observed the largest increase in fecundity when both the male and female were treated with antibiotics which suggests that, however the deleterious effect is mediated, it 
can be horizontally transferred from males to females, potentially via the regurgitated drop. This could explain why females treated with antibiotics mated to normal males still exhibited suppressed fecundity. It is worth noting here that there is potential for some bacterial transfer during the mating trials from flies that have intact gut bacteria, to individuals with suppressed gut bacteria. However, this is unlikely to affect the results as the time taken to conduct all experiments is not sufficient for the gut bacteria to be re-colonised (e.g. Sang et al., 1956).

The fact that females more readily mated with normally-fed males when they themselves lacked gut bacteria is similar to the effect seen in Immonen et al.'s (2009) study, in which starved females would prefer nuptial gifts from males that were "well fed". However, in order to further explore this idea, more in depth study on how exactly the nuptial gift factors into this system is needed. These studies could examine the effect of removing gut bacteria on the bacterial content of the gift itself, measure drop size and quantity, and examine whether female behaviour is altered by any of these variables. However, as the nuptial gift is very small and only produced at the time of mating, it would be very difficult to do this in practise, and a very robust experimental design would be needed.

In conclusion, this study has demonstrated that gut bacteria influences female preference and may lower female fecundity in D. subobscura, providing more evidence for the growing consensus that gut bacteria can play a role in the mating behaviour of a host. Our study also suggests that cues that indicate the bacterial composition of the gut can be detected by potential mates, which may be even more important within mating systems that involve nuptial giftgiving, if the gift is partially determined by the nuptial gift contents as our study suggests. Increasingly we are coming to understand the wide ranging impacts gut-associated bacteria have on the biology of the host.

ACKNOWLEDGEMENTS. The authors would like to thank G. Lawson, T. Heyes, and R. Verspoor for their technical assistance, and A. Lizé, T. Price, and G. Hurst for their comments on the manuscript.

\section{REFERENCES}

Albo M.J., Bilde T. \& Uhl G. 2013: Sperm storage mediated by cryptic female choice for nuptial gifts. - Proc. R. Soc. Lond. (B) 280: 20131735, 6 pp.

Bacigalupe L.D., Crudgington H.S., Hunter F., Moore A.J. \& SNoOK R.R. 2007: Sexual conflict does not drive reproductive isolation in experimental populations of Drosophila pseudoobscura. - J. Evol. Biol. 20: 1763-1771.

Ben-Yosef M., Jurkevitch E. \& Yuval B. 2008: Effect of bacteria on nutritional status and reproductive success of the Mediterranean fruit fly Ceratitis capitata. - Physiol. Entomol. 3: $145-154$

BRUCKer R.M. \& Bordenstein S.R. 2013: The hologenomic basis of speciation: gut bacteria cause hybrid lethality in the genus Nasonia. - Science 341: 667-669.

Debelle A., Ritchie M.G. \& Snook R.R. 2014: Evolution of divergent female mating preference in response to experimental sexual selection. - Evolution 68: 2524-2533.
Drew R.A.I., Courtice A.C. \& Teakle D.S. 1983: Bacteria as a natural source of food for adult fruit flies (Diptera: Tephritidae). - Oecologia 60: 279-284.

Everaerts C., Lacaille F. \& Ferveur J.F. 2010: Is mate choice in Drosophila males guided by olfactory or gustatory pheromones? - Anim. Behav. 79: 1135-1146.

Haine E.R., PICKuP N.J. \& CoOK J.M. 2005: Horizontal transmission of Wolbachia in a Drosophila community. - Ecol. Entomol. 4: 464-472.

Hamm C.A., Begun D.J., Vo A., Smith C.C.R., Saelao P., Shaver A.O., Jaenike J. \& Turelli M. 2014: Wolbachia do not live by reproductive manipulation alone: infection polymorphism in Drosophila suzukii and D. subpulchrella. - Mol. Ecol. 23: 4871-4885.

Harcourt A.H., Harvey P.H., Larson S.G. \& Short R.V. 1981: Testis weight, body weight and breeding system in primates. Nature 293: 55-57.

Hartig F. 2017: DHARMa: Residual Diagnostics for Hierarchical (Multi-Level / Mixed) Regression Models. R Package Version 0.1.5. https://CRAN.R-project.org/package=DHARMa

IBM CORPORATION 2013: IBM SPSS Statistics for Windows, Version 22.0. IBM Corp., Armonk, NY.

Immonen E., Hoikkala A., Kazem A. \& Ritchie M. 2009: When are vomiting males attractive? Sexual selection on conditiondependent nuptial feeding in Drosophila subobscura. - Behav. Ecol. 20: 289-295.

Jennions M.D. \& PASSMORe N.I. 1993: Sperm competition in frogs: testis size and a 'sterile male' experiment on Chiromantis xerampelina (Rhacophoridae). - Biol. J. Linn. Soc. 50: 211-220.

Koukou K., Pavlikaki H., Kilias G., Werren J.H., Bourtzis K. \& Alahiotis S.N. 2006: Influence of antibiotic treatment and Wolbachia curing on sexual isolation among Drosophila melanogaster cage populations. - Evolution 60: 87-96.

KRIMBAS C.B. 1993: Drosophila subobscura: Biology, Genetics and Inversion Polymorphism. Dr Kovac, Hamburg, 395 pp.

LEwIS Z. \& LizÉ A. 2015: Insect behaviour and the microbiome. —Curr. Op. Ins. Sci. 9: 86-90.

Lizé A., McKay R. \& LewIs Z. 2014: Kin recognition in Drosophila: the importance of ecology and gut microbiota. - Internat. Soc. Micro. Ecol. J. 8: 469-477.

Mazzetto F., Gonella E. \& Alma A. 2015: Wolbachia infection affects female fecundity in Drosophila suzukii. - Bull. Insectol. 68: 153-157.

Pradel E., Zhang Y., Pujol N., Matsuyama T., Bargmann C.I. \& EwBanK J.J. 2007: Detection and avoidance of a natural product from the pathogenic bacterium Serratia marcescens by Caenorhabditis elegans. - Proc. Natn. Acad. Sci. USA 104: 2295-2300.

Price T.A.R., Lizé A., Marcello M. \& Bretman A. 2012: Experience of mating rivals causes males to modulate sperm transfer in the fly Drosophila pseudoobscura. - J. Insect Physiol. 58: 1669-1675.

R Core Team 2015: $R$ : A Language and Environment for Statistical Computing. R Foundation for Statistical Computing, Vienna.

Ridley E.V., Wong A.C., Westmiller S. \& Douglas A.E. 2012: Impact of the resident microbiota on the nutritional phenotype of Drosophila melanogaster. - PLoS ONE 7(5): e36765, 7 pp.

Rosengaus R.B., Zecher C.N., Shultheis K.F., Bruker R.M. \& BoRDENSTEIN S.R. 2011: Disruption of the termite gut microbiota and its prolonged consequences for fitness. - Appl. Environ. Microbiol. 77: 4303-4312.

SANG J.H. 1956: The quantitative nutritional requirements of Drosophila melanogaster. - J. Exp. Biol. 33: 45-72. 
Scott D., Shields A., Straker M., Dalrymple H., Dhillon P.K. \& HARBINDER S. 2011: Variation in the male pheromones and mating success of wild caught Drosophila melanogaster. PLOS ONE 6(8): e23645, 8 pp.

Sharon G., Segal D., Ringo J.M., Hefetz A., Zilver-Rosenbero I. \& Rosenberg E. 2010: Commensal bacteria play a role in mating preference of Drosophila melanogaster. - Proc. Natn. Acad. Sci. USA 107: 20051-20056.

Sharon G., Segal D., Zilber-Rosenberg I. \& Rosenberg E. 2011: Symbiotic bacteria are responsible for diet-induced mating preference in Drosophila melanogaster, providing support for the hologenome concept of evolution. - Gut Microb. 2: 190-192.
Thornhill R. \& Alcock J. 1983: The Evolution of Insect Mating Systems. Harvard University Press, Harvard, $547 \mathrm{pp}$.

Verspoor R., Cuss M. \& Price T.A.R. 2015: Age-based mate choice in the monandrous fruit fly Drosophila subobscura. Anim. Behav. 102: 199-207.

Wong A.C., Dobson A.J. \& Douglas A.E. 2014: Gut microbiota dictates the metabolic response of Drosophila to diet. $-J$. Exp. Biol. 217: 1894-1901.

Zhang Y., Lu H. \& BARGMANN C.I. 2005: Pathogenic bacteria induce aversive olfactory learning in Caenorhabditis elegans. Nature 438: 179-184.

Received August 2, 2017; revised and accepted October 9, 2017 Published online October 23, 2017 